\title{
The Healthcare Cost Burden of Acute Myocardial Infarction in Alberta, Canada
}

\author{
Dat T. Tran ${ }^{1,2}$ - Arto Ohinmaa ${ }^{1,3} \cdot$ Nguyen X. Thanh ${ }^{1,3} \cdot$ Robert C. Welsh ${ }^{2,4,5}$. \\ Padma Kaul ${ }^{2,4}$
}

Published online: 2 November 2017

(c) The Author(s) 2017. This article is an open access publication

\begin{abstract}
Objectives Little is known about the cost burden of acute myocardial infarction (AMI) on healthcare systems. Accordingly, we examined the long-term trends of healthcare costs for AMI in the province of Alberta, Canada.

Methods We linked five Albertan health databases, including ambulatory care, hospitalization, practitioner claims, pharmaceutical information network, and population registry to identify patients with a primary diagnosis of AMI between 2004 and 2013. We used the Alberta Interactive Health Data Application to provide unit costs for ambulatory care and inpatient services, claim paid amounts for physician services, and the Alberta Drug Benefit List for drug prices. Healthcare costs for AMI were grouped into ambulatory care, hospitalization, physician costs, and drug costs. All costs were converted to 2016 Canadian dollar values $(\$ \mathrm{Can})$.
\end{abstract}

Electronic supplementary material The online version of this article (https://doi.org/10.1007/s41669-017-0061-0) contains supplementary material, which is available to authorized users.

\section{Dat T. Tran}

dttran@ualberta.ca

1 School of Public Health, University of Alberta, Edmonton, $\mathrm{AB}$, Canada

2 Canadian VIGOUR Centre, University of Alberta, 2-132 Li Ka Shing Centre, Edmonton, AB, Canada

3 Institute of Health Economics, Edmonton, AB, Canada

4 Department of Medicine, University of Alberta, Edmonton, $\mathrm{AB}$, Canada

5 Mazankowski Alberta Heart Institute, University of Alberta Hospital, Edmonton, Canada
Results A total of 52,912 patients with AMI were included in the analysis. Patient age decreased over time, as did the proportion of females. AMI cost the Alberta healthcare system Can $\$ 1033$ million during the study period; of which the largest proportion was hospitalization costs (Can\$716.4 million, 63.1\%), followed by drug costs (Can $\$ 147.2$ million, 21.1\%), ambulatory care costs (Can $\$ 94.5$ million, $8.8 \%)$ and physician costs (Can $\$ 74.9$ million, $7.0 \%)$. The cost per AMI hospitalization decreased from Can $\$ 14,116$ in 2004 to Can\$11,792 in $2013(p<0.001)$.

Conclusions Healthcare costs for AMI are significant; however, they decreased slightly during the study period. Hospital services accounted for the largest share of the costs. There are opportunities for further savings in AMI care.

\section{Key Points for Decision Makers}

Healthcare cost burden of acute myocardial infarction (AMI) is high and hospital services accounted for the largest proportion of healthcare costs for AMI patients.

Compared with other MI groups, Non-ST segment elevation MI is the biggest cost driver.

Healthcare costs for AMI decreased during the study period in the province of Alberta, Canada despite an increase in health service utilization among AMI patients. Further investigation is needed to better understand the trends and to explore replication of the factors that contributed to a decrease in AMI healthcare costs in other jurisdictions. 


\section{Introduction}

Acute myocardial infarction (AMI) is an acute condition requiring expedited diagnosis and intervention. It is the leading cause of morbidity and mortality in many parts of the world [1] and accounts for half of the 17 million worldwide annual deaths from cardiovascular disease (CVD) [2]. Hospital readmission is common among patients with AMI [3]. It is estimated that $37 \%$ of the US population were living with CVD in 2010 and the direct medical costs for CVD are predicted to triple from US\$272.5 billion in 2010 to US\$818.1 billion in 2030, while the productivity losses are projected to increase $61 \%$ during the same period [4]. In Canada, CVD is the second most expensive healthcare burden, costing Can $\$ 22$ billion in 2000 ( Can $\$ 30$ billion in 2016 dollars) [5]. The higher prevalence of AMI associated with an aging population [6] and improved survival among patients with AMI [7] will likely increase its cost burden over time. However, little is known about the current cost burden of AMI on the healthcare systems, and how this cost burden is changing over time. In this study, we examined the healthcare costs associated with AMI between 2004 and 2013 in the province of Alberta, Canada.

\section{Methods}

\subsection{Data Source and Study Population}

We conducted a retrospective cohort study using five linked administrative databases including Ambulatory Care Database, acute care inpatient records (Discharge Abstract Database [DAD]), Practitioner Claims, Pharmaceutical Information Network (PIN), and Population Registry in the province of Alberta, Canada [8]. Ambulatory Care Database contains all ambulatory care utilization (same-day surgery, day procedures, emergency department visits, and community rehabilitation services at publicly funded facilities). It provides information on patient demographics, diagnoses, procedures, and Comprehensive Ambulatory Classification System (CACS) grouping identifying homogenous patient clusters [9]. DAD tracks all acute-care hospitalizations in the province. It contains patient demographics, diagnoses, and Case Mix Group (CMG) classification (equivalent to CACS for hospitalized patients) [10]. Practitioner Claims contains fee-for-service claims information for physicians and other providers for insured health services. PIN records information on drugs dispensed for prescribed medications at almost all pharmacies in the provinces. Finally, Population Registry provide demographic and vital statistics for all inhabitants of
Alberta [8]. The annual population of Alberta during 2004-2013 was used to calculate AMI prevalence over time [11].

We first selected all ambulatory care visits and hospitalizations from April 1, 2004 to March 31, 2014 (fiscal years [FY] 2004-2013) in which AMI (International Classification of Diseases [ICD], 10th revision, codes I21 or I22) was recorded as the primary diagnosis to create the study cohort. All practitioner claims relating to cohort members with a primary diagnosis of AMI (ICD, 9th revision, code 410) and AMI-related drug dispensing events to cohort members that occurred during the study period were then retrieved. AMI-related drugs were defined as (1) antihypertensive agents, (2) diuretics, (3) peripheral vasodilators, (4) $\beta$-blockers, (5) calcium channel blockers, (6) agents acting on the renin-angiotensin system, (7) lipidmodifying agents, and (8) antithrombotic agents. Anatomical Therapeutic Chemical (ATC) drug codes are presented in Supplemental Table S1 in the electronic supplementary material.

\subsection{Main Outcomes}

The main outcomes were total and annual costs for AMI. From a healthcare payer perspective, we defined annual costs as the annual summation of hospitalization, ambulatory care, practitioner claims, and drug costs incurred for all patients with AMI as the primary diagnosis. Other outcomes were distribution of healthcare costs by MI subtypes (ST-segment elevation MI [STEMI], non-STsegment elevation MI [NSTEMI], and undefined/recurrent MI [Other MI]), and by sex and age $(<50,50-59,60-69$, $70-79$, and $\geq 80$ years). We also examined distribution of hospitalization costs by revascularization procedures (percutaneous coronary intervention $[\mathrm{PCI}]$ ) and coronary artery bypass grafting $[\mathrm{CABG}])$.

\subsection{Hospitalization Costs}

We used the Alberta Interactive Health Data Application (AIHDA) Hospital Inpatient Care Case Costs version 2013 to provide dollar values for each hospitalization based on its CMG classification [12]. The AIHDA CMG costs provide functional center direct (e.g., nursing, diagnostic, and therapeutic costs) and indirect costs (e.g., general administration and support services) [13]. As the CMG codes were only available for FYs 2006-2012 in the DAD, we developed a generalized linear model (GLM) with gamma distribution and log link to estimate the average costs per hospitalization during this time period using a previously described algorithm [14]. The variables included in the model were FY, MI subtypes, patient sex and age group. 
We used the model's coefficients to calculate average annual costs per hospitalization for FYs 2004, 2005, and 2013 for which CMG codes were not available. Annual AMI hospitalization costs for each combination of sex, age group, and MI subtype were derived by multiplying number of hospitalizations in each group by the costs per hospitalization in that group.

\subsection{Ambulatory Care Costs}

We used the AIHDA Ambulatory Care Case Costs version 2013 to provide dollar values for each ambulatory care visit based on its CACS code [12]. Similar to AIHDA CMG costs, the AIHDA CACS costs provide functional center direct and indirect costs [15]. The CACS codes were only available for FYs 2006-2012 in the ambulatory care database. Therefore, we also developed a GLM model with gamma distribution and log link with FY, MI subtypes, patient sex and age group being independent variables to estimate the average costs per ambulatory visit during this time period and used the model's coefficients to calculate average costs per ambulatory care visits for FYs 2004, 2005, and 2013, for which the CACS codes were not available. Annual AMI ambulatory care costs for each combination of sex, age group, and MI subtype were derived by multiplying the number of visits in each group by the costs per visit in that group.

\subsection{Practitioner Claims Costs}

The practitioner claims database provides a paid amount and a system assessed amount to each claim. We used the paid amounts as costs for fee-for-service claims. For alternative relationship plan (ARP) claims $(\sim 10 \%$ of all claims) where the paid amounts were unavailable, the system assessed amounts were used instead. We summed all claim costs in a year to determine annual practitioner claims costs.

\subsection{Drug Costs}

The drug data were only available from FY 2008-2013. We used the Alberta Drug Benefit List (ADBL) [16] to provide drug unit prices. If a drug price was not listed in the ADBL, we used the market price at Canada Drugs [17]. Drug costs of a dispensing event were then derived by multiplying the price of a dispensed unit by the number of units dispensed. We summed the costs of individual dispensing events per year to determine annual drug costs.

\subsection{Statistical Analysis}

We summarized patient characteristics using means ( \pm $\mathrm{SD})$, medians (interquartile ranges), counts and percentages, as appropriate. A patient was counted each year if s/he had either a hospitalization or an ambulatory care visit or a practitioner claim during that year. As there is no indication of MI subtype in the practitioner claims and PIN databases, we assumed the MI subtype of the previous ambulatory care visit or hospitalization, whichever was closest, for a claim or drug dispensing event. We used previously validated ICD codes to identify patient comorbidities [18]. Comorbidities were considered to be present if they were recorded in any hospitalization or ambulatory care visit in each year of the study period. We used the Canadian Classification of Health Interventions [19] to identify PCI (codes of 1.IJ.50** and 1.IJ.57.GQ**) and CABG (code of 1.IJ.76**) in any of 20 intervention fields in the DAD record. Univariate GLM regression was used to test for trends of costs over time. A non-parametric trend test was used for trend tests of medians, and univariate linear regression was used for trend tests of means. All costs were converted to Canadian 2016 dollar values using the Bank of Canada inflation calculator [20].

All analyses were performed using Stata version 14 (Stata Corporation, College Station, TX, USA); Two-sided $p$ values $<0.05$ were considered statistically significant. The study was approved by the Health Research Ethics Board at the University of Alberta (IRB number: Pro00066424).

\section{Results}

\subsection{Study Cohort Descriptive Statistics}

Between FY 2004 and 2013, there were 55,384 hospitalizations, 75,309 ambulatory-care visits, 524,238 practitioner claims, and 4,798,869 drug-dispensing events involving 52,912 unique patients with AMI (Table 1). Patient characteristics by year are presented in Table 2 . The number of patients increased from 6031 in 2004 to 7455 in $2013(p<0.001)$, as did the number of hospitalizations (2004: 4935; 2013: 6071; $p<0.001)$. However, population prevalence rates remained stable $(p=0.782)$. The mean age of patients decreased over time $(p<0.001)$, as did the proportion of females $(p<0.001)$. The median hospital length of stay (LOS) decreased from 7 days in 2004 to 5 days in $2013(p<0.001)$. Heart failure and diabetes mellitus were the most common comorbidities. The rates of heart failure decreased (2004: 16.4\%; 2013: 
Table 1 Health services utilization for patients with acute myocardial infarction (AMI) in Alberta, Canada, 2004-2013

\begin{tabular}{|c|c|c|c|c|c|c|c|c|c|c|c|}
\hline Variable & Total & 2004 & 2005 & 2006 & 2007 & 2008 & 2009 & 2010 & 2011 & 2012 & 2013 \\
\hline $\begin{array}{l}\text { Hospital } \\
\quad \text { admissions, } N\end{array}$ & 55,384 & 4935 & 4978 & 4856 & 5650 & 5429 & 5670 & 5875 & 5891 & 6029 & 6071 \\
\hline STEMI, $n(\%)$ & $\begin{array}{r}18,579 \\
(33.6)\end{array}$ & $\begin{array}{l}1560 \\
(31.6)\end{array}$ & $\begin{array}{l}1527 \\
(30.7)\end{array}$ & $\begin{array}{l}1594 \\
(32.8)\end{array}$ & $\begin{array}{l}1970 \\
(34.9)\end{array}$ & $\begin{array}{l}1795 \\
(33.1)\end{array}$ & $\begin{array}{l}1942 \\
(34.3)\end{array}$ & $\begin{array}{l}2140 \\
\quad(36.4)\end{array}$ & $\begin{array}{l}2028 \\
(34.4)\end{array}$ & $\begin{array}{l}2005 \\
(33.3)\end{array}$ & $\begin{array}{l}2018 \\
(33.2)\end{array}$ \\
\hline NSTEMI, $n(\%)$ & $\begin{array}{r}30,362 \\
(54.8)\end{array}$ & $\begin{array}{l}2161 \\
\quad(43.8)\end{array}$ & $\begin{array}{l}2470 \\
\quad(49.6)\end{array}$ & $\begin{array}{l}2456 \\
(50.6)\end{array}$ & $\begin{array}{l}3151 \\
\quad(55.8)\end{array}$ & $\begin{array}{l}3145 \\
\quad(57.9)\end{array}$ & $\begin{array}{l}3209 \\
\quad(56.6)\end{array}$ & $\begin{array}{r}3290 \\
\quad(56)\end{array}$ & $\begin{array}{l}3408 \\
(57.9)\end{array}$ & $\begin{array}{l}3547 \\
\quad(58.8)\end{array}$ & $\begin{array}{l}3525 \\
(58.1)\end{array}$ \\
\hline Other MI, $n(\%)$ & $\begin{array}{l}6443 \\
(11.6)\end{array}$ & $\begin{array}{l}1214 \\
(24.6)\end{array}$ & $\begin{array}{l}981 \\
(19.7)\end{array}$ & $\begin{array}{l}806 \\
(16.6)\end{array}$ & $\begin{array}{l}529 \\
(9.4)\end{array}$ & $\begin{array}{l}489 \\
\quad(9.0)\end{array}$ & $\begin{array}{l}519 \\
(9.2)\end{array}$ & $\begin{array}{l}445 \\
\quad(7.6)\end{array}$ & $\begin{array}{l}455 \\
(7.7)\end{array}$ & $\begin{array}{l}477 \\
\quad(7.9)\end{array}$ & $\begin{array}{l}528 \\
\quad(8.7)\end{array}$ \\
\hline $\begin{array}{l}\text { Ambulatory care } \\
\text { visits, } N\end{array}$ & 75,309 & 7590 & 6674 & 6417 & 6534 & 8938 & 9624 & 10,709 & 8101 & 5242 & 5480 \\
\hline STEMI, $n(\%)$ & $\begin{array}{r}13,929 \\
(18.5)\end{array}$ & $\begin{array}{l}1768 \\
(23.3)\end{array}$ & $\begin{array}{l}1389 \\
(20.8)\end{array}$ & $\begin{array}{l}1230 \\
(19.2)\end{array}$ & $\begin{array}{l}1106 \\
(16.9)\end{array}$ & $\begin{array}{l}1170 \\
(13.1)\end{array}$ & $\begin{array}{l}1213 \\
(12.6)\end{array}$ & $\begin{array}{l}1459 \\
(13.6)\end{array}$ & $\begin{array}{l}1603 \\
(19.8)\end{array}$ & $\begin{array}{l}1452 \\
(27.7)\end{array}$ & $\begin{array}{l}1539 \\
(28.1)\end{array}$ \\
\hline NSTEMI, $n(\%)$ & $\begin{array}{r}19,985 \\
(26.5)\end{array}$ & $\begin{array}{l}2297 \\
\quad(30.3)\end{array}$ & $\begin{array}{l}2042 \\
\quad(30.6)\end{array}$ & $\begin{array}{l}2062 \\
(32.1)\end{array}$ & $\begin{array}{l}1549 \\
(23.7)\end{array}$ & $\begin{array}{r}1784 \\
(20)\end{array}$ & $\begin{array}{l}1780 \\
(18.5)\end{array}$ & $\begin{array}{l}1944 \\
\quad(18.2)\end{array}$ & $\begin{array}{l}1937 \\
(23.9)\end{array}$ & $\begin{array}{l}2249 \\
(42.9)\end{array}$ & $\begin{array}{l}2341 \\
(42.7)\end{array}$ \\
\hline Other MI, $n(\%)$ & $\begin{array}{r}41,395 \\
\quad(55.0)\end{array}$ & $\begin{array}{l}3525 \\
\quad(46.4)\end{array}$ & $\begin{array}{l}3243 \\
\quad(48.6)\end{array}$ & $\begin{array}{l}3125 \\
(48.7)\end{array}$ & $\begin{array}{l}3879 \\
(59.4)\end{array}$ & $\begin{array}{l}5984 \\
(67)\end{array}$ & $\begin{array}{l}6631 \\
(68.9)\end{array}$ & $\begin{array}{l}7306 \\
(68.2)\end{array}$ & $\begin{array}{l}4561 \\
\quad(56.3)\end{array}$ & $\begin{array}{l}1541 \\
(29.4)\end{array}$ & $\begin{array}{l}1600 \\
(29.2)\end{array}$ \\
\hline $\begin{array}{l}\text { Practitioner claims, } \\
\quad N\end{array}$ & 524,238 & 35,055 & 42,511 & 46,866 & 52,067 & 50,815 & 54,964 & 55,261 & 56,845 & 63,497 & 66,357 \\
\hline STEMI, $n(\%)$ & $\begin{array}{r}164,346 \\
(31.4)\end{array}$ & $\begin{array}{l}8242 \\
(23.5)\end{array}$ & $\begin{array}{r}10,146 \\
(23.9)\end{array}$ & $\begin{array}{c}11,728 \\
(25)\end{array}$ & $\begin{array}{r}16,417 \\
(31.5)\end{array}$ & $\begin{array}{r}15,372 \\
(30.3)\end{array}$ & $\begin{array}{r}18,307 \\
(33.3)\end{array}$ & $\begin{array}{r}19,477 \\
(35.2)\end{array}$ & $\begin{array}{r}20,232 \\
(35.6)\end{array}$ & $\begin{array}{r}21,497 \\
(33.9)\end{array}$ & $\begin{array}{r}22,928 \\
(34.6)\end{array}$ \\
\hline NSTEMI, $n(\%)$ & $\begin{array}{r}248,476 \\
(47.4)\end{array}$ & $\begin{array}{r}13,800 \\
(39.4)\end{array}$ & $\begin{array}{r}19,275 \\
(45.3)\end{array}$ & $\begin{array}{r}21,820 \\
(46.6)\end{array}$ & $\begin{array}{r}25,293 \\
(48.6)\end{array}$ & $\begin{array}{c}25,420 \\
(50)\end{array}$ & $\begin{array}{r}26,238 \\
(47.7)\end{array}$ & $\begin{array}{r}25,302 \\
(45.8)\end{array}$ & $\begin{array}{r}27,098 \\
(47.7)\end{array}$ & $\begin{array}{r}32,127 \\
(50.6)\end{array}$ & $\begin{array}{c}32,103 \\
(48.4)\end{array}$ \\
\hline Other MI, $n(\%)$ & $\begin{array}{r}111,416 \\
(21.3)\end{array}$ & $\begin{array}{c}13,013 \\
(37.1)\end{array}$ & $\begin{array}{r}13,090 \\
(30.8)\end{array}$ & $\begin{array}{r}13,318 \\
(28.4)\end{array}$ & $\begin{array}{r}10,357 \\
(19.9)\end{array}$ & $\begin{array}{r}10,023 \\
(19.7)\end{array}$ & $\begin{array}{c}10,419 \\
(19)\end{array}$ & $\begin{array}{c}10,482 \\
(19)\end{array}$ & $\begin{array}{l}9515 \\
(16.7)\end{array}$ & $\begin{array}{l}9873 \\
\quad(15.5)\end{array}$ & $\begin{array}{r}11,326 \\
(17.1)\end{array}$ \\
\hline $\begin{array}{l}\text { Drug dispensing } \\
\text { events, } N\end{array}$ & $4,798,869$ & & & & & 636,997 & 699,885 & 762,841 & 859,446 & 888,912 & 950,788 \\
\hline STEMI, $n(\%)$ & $\begin{array}{c}1,140,622 \\
(23.8)\end{array}$ & & & & & $\begin{array}{r}138,299 \\
(21.7)\end{array}$ & $\begin{array}{r}157,887 \\
(22.6)\end{array}$ & $\begin{array}{r}177,750 \\
(23.3)\end{array}$ & $\begin{array}{r}204,535 \\
(23.8)\end{array}$ & $\begin{array}{r}220,393 \\
(24.8)\end{array}$ & $\begin{array}{r}241,758 \\
(25.4)\end{array}$ \\
\hline NSTEMI, $n(\%)$ & $\begin{array}{l}2,487,284 \\
\quad(51.8)\end{array}$ & & & & & $\begin{array}{r}340,984 \\
(53.5)\end{array}$ & $\begin{array}{r}367,292 \\
(52.5)\end{array}$ & $\begin{array}{r}392,599 \\
(51.5)\end{array}$ & $\begin{array}{r}440,728 \\
(51.3)\end{array}$ & $\begin{array}{r}455,753 \\
(51.3)\end{array}$ & $\begin{array}{r}489,928 \\
(51.5)\end{array}$ \\
\hline Other MI, $n(\%)$ & $\begin{array}{l}1,170,963 \\
(24.4)\end{array}$ & & & & & $\begin{array}{r}157,714 \\
(24.8)\end{array}$ & $\begin{array}{l}174,706 \\
(25)\end{array}$ & $\begin{array}{r}192,492 \\
(25.2)\end{array}$ & $\begin{array}{r}214,183 \\
(24.9)\end{array}$ & $\begin{array}{r}212,766 \\
(23.9)\end{array}$ & $\begin{array}{l}219,102 \\
(23)\end{array}$ \\
\hline $\begin{array}{l}\text { Hospital LOS in } \\
\text { days, median } \\
\text { (IQR) }\end{array}$ & & $7(4-10)$ & $6(4-10)$ & $6(4-9)$ & $5(4-9)$ & $5(4-8)$ & $5(3-8)$ & $5(3-7)$ & $5(3-7)$ & $5(3-7)$ & $5(3-7)$ \\
\hline
\end{tabular}

$I Q R$ interquartile range, LOS acute length of stay, MI myocardial infarction, NSTEMI non-ST-segment elevation MI, STEMI ST-segment elevation MI

$12.7 \% ; p<0.001)$ while the rates of diabetes mellitus increased over time (2004: 20.1\%; 2013: 29.0\%; $p<0.001)$. However, the median Charlson score remained unchanged during the study period ( $p=0.263$ ).

\subsection{Total Healthcare Costs}

Overall, AMI cost the Alberta healthcare system Can $\$ 1033$ million between 2004 and 2013. Of this, hospitalization costs accounted for the majority (Can\$716.4 million), followed by drug costs (Can $\$ 147.2$ million), ambulatory care costs (Can\$94.5 million), and practitioner claims costs (Can\$74.9 million). Proportionally, hospitalization, drug, ambulatory care, and practitioner claims costs, respectively, accounted for $63.1,21.1,8.8$, and $7.0 \%$ of the total healthcare costs for AMI between FY 2008 and 2013 where data of all four service components were available (Fig. 1).

Both hospitalization (annual average Can\$71.6 million; $p=0.141$ ) and ambulatory care (annual average Can $\$ 9.5$ million; $p=0.888$ ) costs remained unchanged despite a decrease in costs per hospitalization during the study period (2004 Can\$14,116; 2013 Can\$11,792; $p<0.001)$. Practitioner claims costs increased from Can\$5.9 million in 2004 to Can\$9.1 million in $2013(p<0.001)$, primarily due to a $7.6 \%$ annual increase in the number of claims during the study period. In contrast, drug costs dropped from Can\$28.6 million in 2008 to Can\$18.3 million in 
Table 2 Characteristics of study population

\begin{tabular}{|c|c|c|c|c|c|c|c|c|c|c|}
\hline Variable & 2004 & 2005 & 2006 & 2007 & 2008 & 2009 & 2010 & 2011 & 2012 & 2013 \\
\hline Patients, $N$ & 6031 & 6487 & 6741 & 6872 & 6972 & 7102 & 7347 & 7517 & 7419 & 7455 \\
\hline Prevalence rate (/100,000 population) & 186 & 195 & 197 & 196 & 194 & 193 & 197 & 198 & 191 & 186 \\
\hline Female $(\%)$ & 33.3 & 33.8 & 33.3 & 32.6 & 32.6 & 32.3 & 31.0 & 30.9 & 33.0 & 31.2 \\
\hline Age in years, mean (SD) & $\begin{array}{l}67.1 \\
(14.3)\end{array}$ & $\begin{array}{l}67.1 \\
(14.4)\end{array}$ & $\begin{array}{l}66.9 \\
(14.4)\end{array}$ & $\begin{array}{l}66.4 \\
(14.3)\end{array}$ & $\begin{array}{l}66.3 \\
(14.4)\end{array}$ & $\begin{array}{l}66.5 \\
(14.4)\end{array}$ & $\begin{array}{l}66.0 \\
(14.4)\end{array}$ & $\begin{array}{l}66.3 \\
(14.3)\end{array}$ & $\begin{array}{l}66.6 \\
(14.3)\end{array}$ & $\begin{array}{l}66.4 \\
(14.1)\end{array}$ \\
\hline Age in years, median (IQR) & $\begin{array}{l}68 \\
(56-79)\end{array}$ & $\begin{array}{l}68 \\
(56-79)\end{array}$ & $\begin{array}{l}67 \\
(56-78)\end{array}$ & $\begin{array}{l}66 \\
(55-78)\end{array}$ & $\begin{array}{l}66 \\
(55-78)\end{array}$ & $\begin{array}{l}66 \\
(55-78)\end{array}$ & $\begin{array}{l}65 \\
(55-78)\end{array}$ & $\begin{array}{l}66 \\
(56-78)\end{array}$ & $\begin{array}{l}66 \\
(56-78)\end{array}$ & $\begin{array}{l}66 \\
(56-77)\end{array}$ \\
\hline \multicolumn{11}{|l|}{ Patient by age group (\%) } \\
\hline$<50$ years & 12.2 & 11.8 & 12.4 & 12.8 & 12.8 & 12.3 & 12.6 & 12.2 & 11.6 & 11.0 \\
\hline $50-59$ years & 20.3 & 21.0 & 21.0 & 21.7 & 22.2 & 22.0 & 23.0 & 22.2 & 22.3 & 22.6 \\
\hline $60-69$ years & 20.5 & 20.1 & 20.9 & 22.2 & 21.5 & 22.2 & 23.0 & 24.6 & 24.0 & 24.8 \\
\hline $70-79$ years & 23.8 & 24.0 & 23.6 & 22.2 & 22.3 & 21.5 & 20.2 & 19.4 & 20.0 & 20.4 \\
\hline$\geq 80$ years & 23.2 & 23.2 & 22.2 & 21.0 & 21.2 & 22.0 & 21.1 & 21.6 & 22.0 & 21.2 \\
\hline \multicolumn{11}{|l|}{ Selected comorbidity (\%) } \\
\hline Heart failure & 16.4 & 17.5 & 15.0 & 15.2 & 14.5 & 13.8 & 13.3 & 11.5 & 13.0 & 12.7 \\
\hline Peripheral vascular disease & 4.2 & 3.9 & 3.3 & 3.9 & 3.1 & 3.1 & 3.0 & 2.8 & 3.1 & 2.9 \\
\hline CVD & 3.3 & 2.7 & 2.7 & 3.2 & 2.4 & 2.2 & 2.2 & 2.2 & 1.9 & 1.5 \\
\hline Cancer & 2.4 & 2.2 & 2.6 & 2.5 & 2.5 & 2.3 & 2.2 & 2.1 & 2.6 & 2.3 \\
\hline COPD & 9.7 & 10.8 & 8.8 & 9.7 & 9.7 & 9.6 & 9.0 & 9.5 & 9.5 & 8.4 \\
\hline Diabetes mellitus & 20.1 & 21.9 & 22.8 & 26.7 & 24.2 & 24.2 & 23.7 & 25.6 & 27.5 & 29.0 \\
\hline Dementia & 2.9 & 3.2 & 2.8 & 2.9 & 2.7 & 2.7 & 2.7 & 2.8 & 2.9 & 2.3 \\
\hline Renal disease & 7.6 & 8.5 & 6.9 & 7.2 & 7.4 & 5.5 & 4.4 & 4.2 & 5.0 & 4.1 \\
\hline Charlson score, median (IQR) & $1(1-2)$ & $1(1-2)$ & $1(1-2)$ & $1(1-2)$ & $1(1-2)$ & $1(1-2)$ & $1(1-2)$ & $1(1-2)$ & $1(1-2)$ & $1(1-2)$ \\
\hline
\end{tabular}

$C O P D$ chronic obstructive pulmonary diseases, $C V D$ cerebrovascular disease, $I Q R$ interquartile range, $L O S$ acute length of stay, $S D$ standard deviation

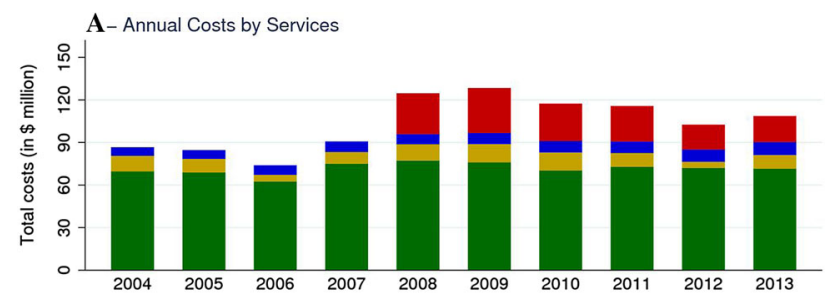

B - Distribution of Costs by Services
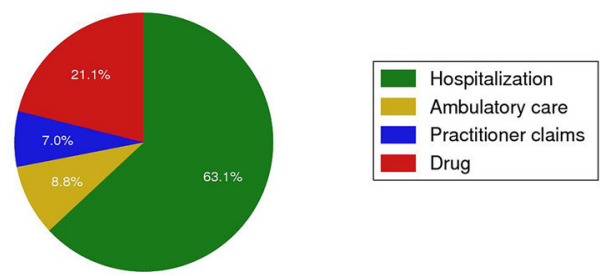

Fig. 1 Healthcare costs for AMI in Alberta, Canada in 2016 Canadian dollars, 2004-2013

$2013(p<0.001)$, despite a constant increase of annual drug dispensing events (Table 1). As a result, annual healthcare costs for AMI decreased from Can\$124.6 million in 2008 to Can\$108.6 million in $2013(p=0.002)$.

\subsection{Distribution of Costs by Myocardial Infarction Subtype, Sex and Age, and Drug Class}

Overall, care for STEMI was more costly than it was for other MI subtypes in both hospital and ambulatory care settings (Table 3). However, since NSTEMI occurred more frequently, it accounted for more than half of the total healthcare costs. While the proportion of STEMI costs $(34.2 \%)$ remained stable during the study period $(p=0.921)$, the NSTEMI contribution increased from $44.2 \%$ in 2004 to $54.7 \%$ in 2013 ( $p=0.006$ ) and other MI costs decreased from $21.5 \%$ in 2004 to $10.8 \%$ in 2013 ( $p=0.006$ ) (Fig. 2). A detailed distribution of the costs by MI subtype in hospitalization, ambulatory care, practitioner claims, and drug domains are presented in Supplemental Figure 1 (see electronic supplementary material).

The total AMI healthcare cost for males was double that for females. Elderly groups (aged $\geq 70$ years) accounted for more than half of total costs for females, but only $34.1 \%$ for males. In contrast, the youngest group (aged $<50$ years) accounted for $14.1 \%$ of total costs for males, about two times higher than their female counterparts (Table 4). 
Table 3 Hospitalization and ambulatory care costs for acute myocardial infarction (AMI) in Alberta, Canada in 2016 Canadian dollars, 2004-2013

\begin{tabular}{|c|c|c|c|c|c|c|c|c|c|c|c|}
\hline Variable & All & 2004 & 2005 & 2006 & 2007 & 2008 & 2009 & 2010 & 2011 & 2012 & 2013 \\
\hline \multicolumn{12}{|l|}{ All hospitalizations } \\
\hline Per hospitalization, Can\$ & 12,935 & 14,116 & 13,858 & 12,886 & 13,284 & 14,219 & 13,429 & 11,958 & 12,354 & 11,963 & 11,792 \\
\hline Total costs, Can $\$$ million & 716.4 & 69.7 & 69.0 & 62.6 & 75.1 & 77.2 & 76.1 & 70.3 & 72.8 & 72.1 & 71.6 \\
\hline \multicolumn{12}{|l|}{ STEMI } \\
\hline Per hospitalization, Can\$ & 13,813 & 15,324 & 14,998 & 14,382 & 13,839 & 14,920 & 14,550 & 12,514 & 13,141 & 12,891 & 12,553 \\
\hline Total costs, Can $\$$ million & 256.6 & 23.9 & 22.9 & 22.9 & 27.3 & 26.8 & 28.3 & 26.8 & 26.6 & 25.8 & 25.3 \\
\hline \multicolumn{12}{|l|}{ NSTEMI } \\
\hline Per hospitalization, Can\$ & 12,702 & 14,147 & 13,814 & 12,564 & 13,223 & 14,103 & 13,015 & 11,823 & 12,117 & 11,627 & 11,600 \\
\hline Total costs, Can $\$$ million & 385.7 & 30.6 & 34.1 & 30.9 & 41.7 & 44.4 & 41.8 & 38.9 & 41.3 & 41.2 & 40.9 \\
\hline \multicolumn{12}{|l|}{ Other MI } \\
\hline Per hospitalization, Can\$ & 11,497 & 12,509 & 12,195 & 10,906 & 11,583 & 12,398 & 11,796 & 10,281 & 10,625 & 10,569 & 10,170 \\
\hline Total costs, Can $\$$ million & 74.1 & 15.2 & 12.0 & 8.8 & 6.1 & 6.1 & 6.1 & 4.6 & 4.8 & 5.0 & 5.4 \\
\hline \multicolumn{12}{|l|}{ All ambulatory care visits } \\
\hline Per visit, Can\$ & 1255 & 1450 & 1424 & 712 & 1273 & 1302 & 1323 & 1198 & 1226 & 832 & 1758 \\
\hline Total costs, Can $\$$ million & 94.5 & 11.0 & 9.5 & 4.6 & 8.3 & 11.6 & 12.7 & 12.8 & 9.9 & 4.4 & 9.6 \\
\hline \multicolumn{12}{|l|}{ STEMI } \\
\hline Per visit, Can\$ & 2363 & 2383 & 2402 & 1126 & 2507 & 3380 & 3551 & 2978 & 2353 & 817 & 2364 \\
\hline Total costs, Can $\$$ million & 32.9 & 4.2 & 3.3 & 1.4 & 2.8 & 4.0 & 4.3 & 4.3 & 3.8 & 1.2 & 3.6 \\
\hline \multicolumn{12}{|l|}{ NSTEMI } \\
\hline Per visit, Can\$ & 2320 & 2379 & 2425 & 908 & 2547 & 3099 & 3599 & 3230 & 2321 & 898 & 2308 \\
\hline Total costs, Can $\$$ million & 46.4 & 5.5 & 5.0 & 1.9 & 3.9 & 5.5 & 6.4 & 6.3 & 4.5 & 2.0 & 5.4 \\
\hline \multicolumn{12}{|l|}{ Other MI } \\
\hline Per visit, Can\$ & 368 & 376 & 374 & 420 & 413 & 361 & 305 & 302 & 364 & 750 & 368 \\
\hline Total costs, Can $\$$ million & 15.2 & 1.3 & 1.2 & 1.3 & 1.6 & 2.2 & 2.0 & 2.2 & 1.7 & 1.2 & 0.6 \\
\hline
\end{tabular}

MI myocardial infarction, NSTEMI non-ST-segment elevation MI, STEMI ST-segment elevation MI

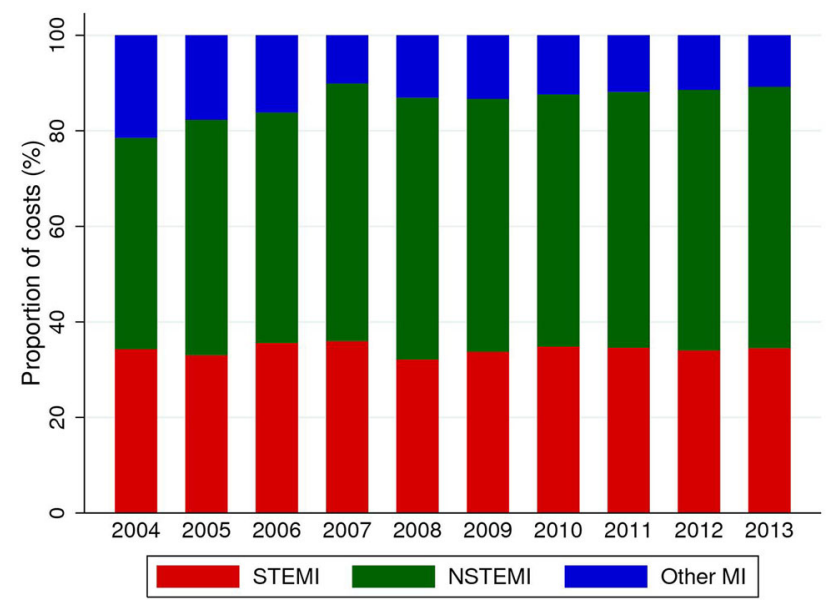

Fig. 2 Distribution of healthcare costs by AMI subtype in Alberta, Canada, 2004-2013. MI myocardial infarction, NSTEMI non-STsegment elevation MI, STEMI ST-segment elevation MI

Lipid-modifying agents, antithrombotic agents, and agents acting on the renin-angiotensin system accounted for the highest drug costs, while diuretics and peripheral vasodilators accounted for the lowest drug costs during the study period (Fig. 3). The annual costs of lipid-modifying agents (2012: Can\$12.7 million; 2013: Can\$5.7 million; $p<0.001)$ and agents acting on the renin-angiotensin system (2012: Can $\$ 5.4$ million; 2013: Can\$3.9 million; $p<0.001)$ dropped gradually over time whereas the annual costs of antithrombotic agents reached a peak of Can\$9.5 million in 2011 and then dropped by half in the following years (Table 5).

\subsection{Distribution of Hospitalization Costs by Revascularization Procedure}

The proportion of hospitalizations with PCI increased from $33.6 \%$ in 2004 to $37.8 \%$ in 2013 ( $p<0.001$ ). In contrast, the proportion of hospitalizations with CABG decreased from $3.0 \%$ in 2004 to $1.1 \%$ in 2013 ( $p<0.001$ ). The cost per hospitalization with CABG (Can\$32,880) was twice as high as the cost per hospitalization with PCI (Can\$14,147; $p<0.001)$. Accordingly, the annual costs for hospitalizations with PCI increased from Can\$24.2 million (34.7\%) in 2004 to Can $\$ 27.8$ million $(38.9 \%)$ in $2013(p=0.012)$, whereas the 
Table 4 Healthcare costs for acute myocardial infarction (AMI) by sex and age in Alberta in 2016 Canadian dollars (millions), 2004-2013

\begin{tabular}{lrrrrr}
\hline Group & Total & Hospitalization & Ambulatory care & Practitioner claims & Drug \\
\hline All patients & 1033.0 & 716.4 & 94.5 & 74.9 & 147.2 \\
Female & 317.5 & 221.6 & 26.9 & 22.2 & 46.9 \\
$<$ 50 years & 23.8 & 17.1 & 2.6 & 1.8 & 2.3 \\
50-59 years & 47.7 & 32.8 & 4.8 & 3.6 & 6.6 \\
60-69 years & 63.6 & 42.5 & 5.9 & 4.6 & 10.6 \\
70-79 years & 81.7 & 56.4 & 6.9 & 5.7 & 12.7 \\
$\geq 80$ years & 100.7 & 72.8 & 6.8 & 6.4 & 14.6 \\
Male & 715.4 & 494.8 & 67.6 & 52.8 & 100.3 \\
$<50$ years & 101.0 & 72.1 & 11.9 & 7.8 & 9.2 \\
50-59 years & 190.0 & 131.3 & 19.9 & 14.6 & 24.2 \\
60-69 years & 180.7 & 121.4 & 17.1 & 13.4 & 28.8 \\
$70-79$ years & 145.6 & 99.5 & 11.9 & 10.3 & 23.9 \\
$\geq 80$ years & 98.1 & 70.5 & 6.8 & 6.6 & 14.2 \\
\hline
\end{tabular}

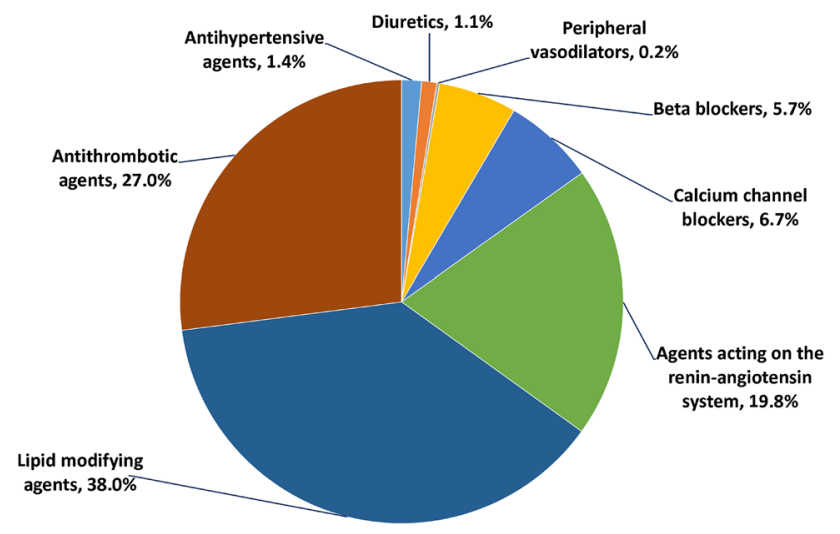

Fig. 3 Drug costs distribution by main Anatomical Therapeutic Classification (ATC) groups

annual costs for hospitalizations with $\mathrm{CABG}$ remained stable during the study period (Can\$2.4 million/year; $p=0.356)$.

\section{Discussion}

Our study of healthcare costs for patients with AMI in the province of Alberta, Canada showed that AMI cost the province approximately Can\$1 billion between 2004 and
2013 (Can $\$ 100$ million per year, equaling $0.4 \%$ of provincial health expenditure in 2013 [21]). Hospital services accounted for the greatest share and practitioner claims accounted for the smallest share of total AMI healthcare costs. During the study period, annual AMI healthcare costs decreased due to a reduction in drug costs and stable hospitalization and ambulatory care costs. NSTEMI was the main cost driver for patients with AMI in the province of Alberta.

In response to ever-increasing healthcare costs, cost containment efforts have been implemented in many countries, including Canada [22]. In addition, there have been significant advances in diagnosis and treatment of AMI in the last several decades, which may lead to productivity improvements in AMI care [23]. For example, there has been a substantial increase in the use of PCI and a decrease in the use of CABG, which is far more costly and resource-intensive than PCI in AMI patients [24-26]. Furthermore, the Canadian Institution for Health Information reports a substantial increase in the generic share of prescriptions in Alberta from 56.6\% in 2008 to $72.1 \%$ in 2013, and this could contribute to the decreased annual drug costs as observed in this study [27]. The combination effect of these factors might explain the decrease in annual
Table 5 Drug costs for acute myocardial infarction (AMI) by Anatomical Therapeutic Classification (ATC) groups in Alberta in 2016 Canadian dollars (thousand), 2004-2013

\begin{tabular}{lrrrrrrr}
\hline ATC group & Total & 2008 & 2009 & 2010 & 2011 & 2012 & 2013 \\
\hline Antihypertensive agents & 2130 & 178 & 258 & 268 & 374 & 492 & 559 \\
Diuretics & 1618 & 229 & 243 & 252 & 284 & 299 & 312 \\
Peripheral vasodilators & 305 & 69 & 61 & 54 & 48 & 37 & 36 \\
B-blockers & 8397 & 1178 & 1299 & 1336 & 1448 & 1522 & 1614 \\
Calcium channel blockers & 9827 & 3023 & 2144 & 1250 & 1214 & 1127 & 1069 \\
Agents acting on the renin-angiotensin system & 29,121 & 5423 & 5777 & 5728 & 4466 & 3798 & 3929 \\
Lipid-modifying agents & 55,956 & 12,655 & 14,720 & 9432 & 7576 & 5854 & 5720 \\
Antithrombotic agents & 39,798 & 5857 & 7075 & 8050 & 9535 & 4226 & 5056 \\
\hline
\end{tabular}


healthcare costs for AMI in Alberta. However, further investigation is needed to better understand this effect.

We found that the per capita cost of AMI in Alberta was Can\$31.7 per capita in 2010 (Can\$117.4 million for a population of 3.7 million [11]). This is lower than the cost of Can\$131.2 per capita in the US [4, 28] (converted to Can\$, 2016 values using the Federal reserve system exchange rate [29] and Bank of Canada inflation calculator [20]). In addition, we observed a downward trend in healthcare costs while Heidenreich et al. forecasted the opposite to happen in the US [4]. This may be due to the fact that the US study included a broader population (AMI plus angina pectoris and other forms of chronic ischemic heart disease). Furthermore, variations in patterns of practice between the two countries may have an impact on medical costs [30, 31], and overall drug prices in the US are approximately 2.9 times higher than in Canada [32].

Our study confirmed that hospital services and drugs account for the majority of total healthcare costs for patients with AMI. While Seo et al. reported that hospitalization costs could account for $73 \%$ of total medical costs for AMI in Korea [33], Mantovani et al. reported a 77 and $15 \%$ attribution of inpatient care and drug costs, respectively, to the total medical costs for AMI in Italy [34]. In addition to opportunities to improve quality of care while controlling costs by preventative strategies [35], these findings emphasize the importance of cost-saving strategies in hospital (i.e., utilizing the right procedure for the right patient and reduction of LOS). During the study period, we observed decreased costs per hospitalization which may be a composite effect of the reduction in costs for patients who underwent PCI [36] and decreased LOS during the study period. It was reported previously that differences in procedure and LOS could contribute 34 and $53 \%$ to the variation in total hospital costs, respectively [31].

Even though STEMI is an acute condition requiring expedited diagnosis and intervention and was more costly to treat, we found that NSTEMI was the biggest resource consumer and cost driver. Our findings are consistent with previously reported results which show a significant increase of the NSTEMI proportion from $52.8 \%$ in 2002 to $68.6 \%$ in 2011 in the US [37]. These findings suggest NSTEMI patients could be a potential target for future cost-containment efforts. Recent studies have shown a high proportion of NSTEMI patients in Canada could be admitted to telemetry wards rather than resource-intensive and costly critical care units with no difference in clinical outcomes [38, 39].

Although our study provides novel data on the healthcare costs of AMI in the province of Alberta, it has some limitations. First, we did not include productivity losses due to mortality and morbidity. Indirect costs for AMI could be equal to or higher than direct medical costs $[4,33]$. Therefore, the true cost burden of AMI on Albertans is substantially higher than what we reported. Second, we did not have drug data for the years 2004-2007, so the drug costs could be significantly higher. Third, we did not include the healthcare costs incurred at privately funded services (e.g., privately funded rehabilitation services). However, these costs should be minor in a universally covered and publicly funded health system like in Alberta. Finally, we could not account for any diagnostic errors and misclassification (in terms of MI subtype). Although Canadian administrative data have been shown to be valid relative to chart abstraction, these data may be affected by data entry errors, omissions, and inconsistencies [40, 41].

\section{Conclusions}

Healthcare costs for AMI are significant; however, they decreased slightly during the study period in the province of Alberta. Not surprisingly, hospital services accounted for the largest proportion of the AMI care costs. There are still opportunities to further improve cost-saving efforts in AMI care. Our study suggests that further investigation is needed to better understand cost-saving efforts in the province of Alberta and to explore the possibility of replication to other jurisdictions.

Author Contributions Dat T. Tran, Arto Ohinmaa, and Padma Kaul conceptualized the study. Dat T. Tran carried out analyses and drafted the manuscript. All of the authors contributed to the writing. All of the authors critically reviewed the manuscript for intellectual content.

\section{Compliance with Ethical Standards}

Data availability statement The data that support the findings of this study are available from Alberta Health; however, restrictions apply to the availability of these data, which were used under license for the current study, and so are not publicly available. Data are, however, available from the authors upon reasonable request and with permission from Alberta Health.

Conflict of interest Dat T. Tran, Robert Welsh, Arto Ohinmaa, Thanh Nguyen, and Padma Kaul declare that they have no conflicts of interest and they received no financial support for this manuscript.

Open Access This article is distributed under the terms of the Creative Commons Attribution-NonCommercial 4.0 International License (http://creativecommons.org/licenses/by-nc/4.0/), which permits any noncommercial use, distribution, and reproduction in any medium, provided you give appropriate credit to the original author(s) and the source, provide a link to the Creative Commons license, and indicate if changes were made. 


\section{References}

1. Turpie AG. Burden of disease: medical and economic impact of acute coronary syndromes. Am J Manag Care. 2006;12(16 Suppl):S430-4.

2. Mendis S, Puska P, Norrving B, World Health Organization., World Heart Federation., World Stroke Organization. Global atlas on cardiovascular disease prevention and control. Geneva: World Health Organization in collaboration with the World Heart Federation and the World Stroke Organization; 2011.

3. Curtis JP, Schreiner G, Wang Y, Chen J, Spertus JA, Rumsfeld JS, Brindis RG, Krumholz HM. All-cause readmission and repeat revascularization after percutaneous coronary intervention in a cohort of medicare patients. J Am Coll Cardiol. 2009;54(10):903-7.

4. Heidenreich PA, Trogdon JG, Khavjou OA, Butler J, Dracup K, Ezekowitz MD, Finkelstein EA, Hong Y, Johnston SC, Khera A, Lloyd-Jones DM, Nelson SA, Nichol G, Orenstein D, Wilson PWF, Woo YJ. Forecasting the future of cardiovascular disease in the united states: a policy statement from the American Heart Association. Circulation. 2011;24:2011.

5. Public Health Agency of Canada. Tracking heart disease and stroke in Canada, 2009. Ottawa: Public Health Agency of Canada; 2009.

6. Statistics Canada. Table 052-0005-estimates of population, by age group and sex for July 1, Canada, provinces and territories. CANSIM (database) [cited 2015 February 28]. http://www5. statcan.gc.ca/cansim/pick-choisir?lang=eng \&p2=33\&id= 0520005 .

7. Tu JV, Nardi L, Fang J, Liu J, Khalid L, Johansen H. Canadian Cardiovascular Outcomes Research T. National trends in rates of death and hospital admissions related to acute myocardial infarction, heart failure and stroke, 1994-2004. CMAJ. 2009;180(13):E118-25.

8. Alberta Health. Overview of Administrative Health Datasets. July 7, 2015 [cited 2016 December 1]. https://open.alberta.ca/dataset/ 657ed26d-eb2c-4432-b9cb-0ca2158f165d/resource/38f47433b33d-4d1e-b959-df312e9d9855/download/Research-HealthDatasets.pdf.

9. Canadian Institute for Health Information. Comprehensive Ambulatory Classification System (CACS). [cited 2017 September 22]. https://www.cihi.ca/en/comprehensiveambulatory-classification-system-cacs.

10. Canadian Institute for Health Information. Case Mix. [cited 2015 July 28]. https://www.cihi.ca/en/data-and-standards/standards/ case-mix.

11. Statistics Canada. Table 051-0001 - Estimates of population, by age group and sex for July 1, Canada, provinces and territories. CANSIM (database) [cited 2015 February 28]. http://www5. statcan.gc.ca/cansim/pick-choisir?lang=eng \&p2=33\&id= 0510001.

12. Alberta Interactive Health Data Application. Interactive Health Data Application. [cited 2016 July 16]. http://www.ahw.gov.ab. ca/IHDA_Retrieval/selectCategory.do.

13. Alberta Health-Health Analytics Branch. Indicator: Hospital Inpatient Case Costing. 2014.

14. Manning WG, Mullahy J. Estimating log models: to transform or not to transform? J Health Econ. 2001;20(4):461-94.

15. Alberta Health-Health Analytics Branch. Indicator: Hospital Ambulatory Care Case Costing. 2014.

16. Alberta Health. Interactive Drug Benefit List. 2016 [cited 2016 September 10]. https://www.ab.bluecross.ca/dbl/publications. html.

17. Canada Drugs. Drug Price. 2016 [cited 2016 September 10]. https://www.canadadrugs.com/.
18. Quan H, Sundararajan V, Halfon P, Fong A, Burnand B, Luthi JC, Saunders LD, Beck CA, Feasby TE, Ghali WA. Coding algorithms for defining comorbidities in ICD-9-CM and ICD-10 administrative data. Med Care. 2005;43(11):1130-9.

19. Canadian Institute for Health Information. Canadian Classification Of Health Interventions. 2012 [cited 2015 February 26]. https://secure.cihi.ca/estore/productSeries.htm?locale=en\&pc= PCC189.

20. Bank of Canada. Inflation Calculator. [cited 2016 August 25]. http://www.bankofcanada.ca/rates/related/inflation-calculator/ ?page_moved $=1$.

21. Canadian Institute for Health Information. National Health Expenditure Trends, 1975 to 2013.

22. Stabile M, Thomson S, Allin S, Boyle S, Busse R, Chevreul K, Marchildon G, Mossialos E. Health care cost containment strategies used in four other high-income countries hold lessons for the United States. Health Aff (Millwood). 2013;32(4):643-52.

23. Reddy K, Khaliq A, Henning RJ. Recent advances in the diagnosis and treatment of acute myocardial infarction. World $\mathbf{J}$ Cardiol. 2015;7(5):243-76.

24. Yeh RW, Sidney S, Chandra M, Sorel M, Selby JV, Go AS. Population trends in the incidence and outcomes of acute myocardial infarction. N Engl J Med. 2010;362(23):2155-65.

25. Tran DT, Welsh RC, Ohinmaa A, Thanh NX, Kaul P. Temporal trends of reperfusion strategies and hospital mortality for patients With STEMI in percutaneous coronary intervention-capable hospitals. Can J Cardiol. 2017;33(4):485-92.

26. Tran DT, Welsh RC, Ohinmaa A, Thanh NX, Bagai A, Kaul P. Quality of acute myocardial infarction care in Canada: a 10-year review of 30-day in-hospital mortality and 30-day hospital readmission. Can J Cardiol. 2017;33(10):1319-26.

27. Canadian Institute for Health Information. Prescribed Frug Spending in Canada, 2013: a focus on public drug programs. 2014 [cited 2017 September 15].

28. U.S. Census Bureau. Population Distribution and Change: 2000 to 2010. 2011.

29. Board of Governors of the Federal Reserve System. Foreign Exchange Rates-H.10. 2017 [cited 2017 February 22]. https:// www.federalreserve.gov/releases/h10/hist/dat00_ca.htm.

30. Mehta RH, Kaul P, Lopes RD, Patel MR, Zheng Y, Pieper KS, Welsh RC, Armstrong PW, Granger CB. Variations in practice and outcomes in patients undergoing primary percutaneous coronary intervention in the United States and Canada: insights from the Assessment of Pexelizumab in Acute Myocardial Infarction (APEX AMI) trial. Am Heart J. 2012;163(5):797-803.

31. Kauf TL, Velazquez EJ, Crosslin DR, Weaver WD, Diaz R, Granger CB, McMurray JJV, Rouleau J-L, Aylward PE, White HD, Califf RM, Schulman KA. The cost of acute myocardial infarction in the new millennium: evidence from a multinational registry. Am Heart J. 2006;151(1):206-12.

32. Patented Medicine Price Review Board Annual Report, 2015. Ottawa. 2016. Report No.: 1495-0561.

33. Seo H, Yoon SJ, Yoon J, Kim D, Gong Y, Kim AR, Oh IH, Kim EJ, Lee YH. Recent trends in economic burden of acute myocardial infarction in South Korea. PLoS One. 2015;10(2):e0117446.

34. Mantovani LG, Fornari C, Madotto F, Riva MA, Merlino L, Ferrario MM, Chiodini V, Zocchetti A, Corrao G, Cesana G. Burden of acute myocardial infarction. Int $\mathrm{J}$ Cardiol. 2011;150(1):111-2.

35. Kahn R, Robertson RM, Smith R, Eddy D. The impact of prevention on reducing the burden of cardiovascular disease. Circulation. 2008;118(5):576-85.

36. Afana M, Brinjikji W, Cloft H, Salka S. Hospitalization costs for acute myocardial infarction patients treated with percutaneous 
coronary intervention in the United States are substantially higher than Medicare payments. Clin Cardiol. 2015;38(1):13-9.

37. Pasterkamp G, den Ruijter HM, Libby P. Temporal shifts in clinical presentation and underlying mechanisms of atherosclerotic disease. Nat Rev Cardiol. 2017;14(1):21-9.

38. van Diepen S, Lin M, Bakal JA, McAlister FA, Kaul P, Katz JN, Fordyce CB, Southern DA, Graham MM, Wilton SB, Newby LK, Granger CB, Ezekowitz JA. Do stable non-ST-segment elevation acute coronary syndromes require admission to coronary care units? Am Heart J. 2016;175:184-92.

39. van Diepen S, Lin M, Ezekowitz JA, McAlister FA, Lee DS, Goodman SG, Armstrong PW, Kaul P. Interprovincial differences in canadian coronary care unit resource use and outcomes. Can J Cardiol. 2017;33(1):166-9.

40. Juurlink DN, Institute for Clinical Evaluative Sciences in Ontario. Canadian Institute for Health Information discharge abstract database a validation study. ICES investigative report. Toronto: Institute for Clinical Evaluative Sciences; 2006. p. 1 online resource (vi, 69 p.).

41. Hinds A, Lix LM, Smith M, Quan H, Sanmartin C. Quality of administrative health databases in Canada: a scoping review. Can J Public Health. 2016;107(1):e56-61. 